\title{
LA FONCTION DE L'ESCAPISME DANS LE ROMAN ALLAH N'EST PAS OBLIGE D'AHMADOU KOUROUMA Messan TOSSA ${ }^{1}$
}

RÉSUMÉ : La figure de l'enfant soldat s'est progressivement établie dans la fiction romanesque africaine centrée sur le récit de la guerre. Du point de vue de l'esthétique de la réception, mais aussi de l'actualité des guerres dans le TiersMonde, le motif de l'enfant soldat fait autorité et trouve une audience confortable auprès des lecteurs. Mais, la mise en scène des enfants dans les récits d'une violence extrême impose des techniques narratives qui tendent à occulter ou du moins à restreindre la cruauté des images présentées au lecteur. Une des stratégies courantes est la fuite de la réalité qui dispense les jeunes protagonistes de décrire les cruautés qu'ils ont commises. L'occlusion des épisodes les plus crus de la narration vise à dépouiller la fiction de la guerre des expériences trop ruineuses pour la psychologie des protagonistes. La présente contribution analyse les segments esthétiques de cet escapisme dans le roman Allah n'est pas obligé d'Ahmadou Kourouma.

MOTS-CLÉS : Enfants-soldats, récit de guerre, escapisme

\footnotetext{
${ }^{1}$ Dr. Messan TOSSA enseigne au Département d'Allemand de l'Université de Lomé depuis 2013. Titulaire d'un Doctorat en Littérature Allemande Contemporaine, il est aussi écrivain d'expression française.
} 


\title{
THE FONCTION OF ESCAPISM IN THE NOVEL ALLAH N'EST PAS OBLIGE OF AHMADOU KOUROUMA
}

\begin{abstract}
The figure of children-soldiers finds a great audience in African novels dealing with war narratives. Because of the actuality of wars in developing countries, the motifs of children-soldiers find an evident interest in literature. But involving children in situations of extreme violence made authors blind some of the very cruel images. One of the most current strategies is to escape the reality, so that the hero must not describe the cruelties he has done. The silence on some episodes of the narrative aims to prevent the protagonist from describing some facts that inevitably charge his psychology. This paper analyses aesthetic segments from this escapism in the novel Allah n'est pas obligé of Ahmadou Kourouma.
\end{abstract}

KEYWORDS : Children-soldiers, war narrative, escapism

Le roman Allah n'est obligé d'Ahmadou Kourouma offre une représentation littéraire saisissante des guerres civiles du Libéria et de la Sierra-Leone. Le spectacle de désintégration des deux Etats est présenté de la perspective d'un enfant-soldat, une focalisation qui impose une approche romanesque complexe. Le décor chaotique de l'action dépasse l'envergure psychique d'un enfant et l'auteur est obligé de déployer des stratégies narratives inédites pour que son narrateur assume les séquences diégétiques du roman. Celui-ci se réfugie dans des paravents esthétiques contre des réalités trop lourdes pour sa psychologie d'enfant.

Le récit de Birahima, est articulé autour d'une ligne déjà tracée par Ken Saro Wiwa dans Soza Boy². Le récit de la guerre impliquant les enfants-soldats répond à la volonté de nombreux auteurs de trouver une audience internationale en traitant d'une thématique récurrente dans la presse internationale. Ainsi s'impose le « politiquement correct » au narrateur de Kourouma qui s'excuse:

Je veux bien m'excuser de vous parler vis-à-vis comme ça. Parce que je ne suis qu'un enfant. Suis dix ou douze ans (il y a deux ans grand-mère disait huit et maman dix) et je parle beaucoup. Un enfant poli écoute, ne garde pas la palabre... Il ne cause pas comme un oiseau gendarme dans les branches de figuier. (KOUROUMA, $2000: 11-12$ )

L'organisation du récit sous-entend la présence d'un interlocuteur, le docteur Mamadou Doumbia qui sollicite le narrateur (KOUROUMA, 2000 : 233) : "Petit Birahima, dis- moi tout ce que tu as vu et fait ; dis - moi comment tout ça s'est pas-

\footnotetext{
${ }^{2}$ Ken Saro-Wiwa : Soza Boy, Longmann African Writers, London, 1994 [1985].
} 
sé ». Birahima y consent et trouve là un vrai exutoire pour amorcer la catharsis ${ }^{3}$ des événements vécus dans ses périples à travers le Libéria et la Sierra-Leone. Symptomatique est le fait que le narrateur garde à l'esprit l'audience de son récit et confirme inconsciemment l'aspect programmatique de la démarche narrative (KOUROUMA, 2000 : 11) : "Il faut expliquer parce que mon blablabla est à lire par toute sorte de gens : des toubabs (toubab signifie blanc) colons, des noirs indigènes sauvages d'Afrique et des francophones de tout gabarit (gabarit signifie genre) ». La nécessité de l'explication est ressentie du point de vue historique, mais aussi psycholinguistique si bien que les refuges du narrateur correspondent à des exigences de clarification historique et psycholinguistique. L'auteur a habilement inséré dans son schéma narratif des paravents historiques et psycholinguistiques qui protègent le narrateur contre la cruauté des images des guerres civiles d'Afrique occidentale en occultant les épisodes nuisibles à son équilibre psychologique.

\section{PARAVENTS HISTORIQUES :}

L'étoffe psychoaffective du narrateur est incompatible avec les clarifications historiques nécessaires à la compréhension du récit. Pourtant, le narrateur se met à expliquer les ressorts historiques de l'action alors que son "école n'est pas arrivée très loin ; jai coupé cours élémentaire deux» (KOUROUMA, 2000 : 10). Dans l'enchaînement des horreurs des guerres civiles libériennes et sierraléonaises, le narrateur déploie sa fiction sur un champ géographique factuel. De là vient la nécessité d'explorer les ressorts politiques, sociaux et historiques qui se sont combinés pour précipiter le décor de l'action dans une situation de désintégration sociopolitique.

La clairvoyance des liens analytiques entre les événements historiques et la situation de guerre civile caractéristique du temps raconté amène à penser à une instance externe qui est tacitement substituée au héros intradiégétique. Le lecteur averti perçoit, au regard de l'âge éventuel du narrateur et de son niveau d'instruction, la présence diffuse d'une obédience narrative capable de relater des décennies d'histoire postcoloniale. La simplicité intellectuelle de Birahima ne peut démêler les enchevêtrements géopolitiques des guerres tribales ouest-africaines qui sont analysées avec une cohérence trop claire pour un enfant de la rue. Ainsi en est-il de la genèse de la guerre civile au Libéria:

C'est pourquoi on dit, les historiens disent que la guerre tribale arriva au Libéria ce soir de Noël 1989. La guerre commença ce 24 décembre 1989, exactement dix ans avant jour pour jour, le coup d'Etat militaire du pays voisin, la Côte

\footnotetext{
${ }^{3}$ Le récit peut revêtir une forme de purification dans le mode de vie Malinké. Voir à cet effet le roman En attendant le vote des bêtes sauvages du même auteur.
} 
d'Ivoire. Depuis cette date, les ennuis pour Samuel Doe allèrent crescendo jusqu'à sa mort. [...] Crescendo jusqu'à sa mort par coupure en tranches. (KOUROUMA, 2000 : 109)

La référence au coup d'Etat militaire de décembre 1999 en Côte d'Ivoire crée une distorsion dans l'échelonnement du temps du récit. Cela sous-entend que l'année mentionnée appartient à l'axe alors très distendu du temps de la narration. L'exactitude des faits historiques mentionnés dans le récit semble consacrer le croisement entre fiction littéraire et vérité historique.

En dehors du fait que ces déviations historiques détournent l'attention du lecteur des spectacles de désolation morbides, elles permettent de situer le décor global de l'action tout en évitant au narrateur de rester cloisonné dans un univers macabre où la violence foncière ruine l'équilibre de l'individu. Se prolonge alors une extraversion platonique où le récit s'attarde exclusivement sur les évènements sans aucune allusion aux effets sur la psychologie des personnages. Le récit prend le pas sur la psychologie des personnages dont l'instinct de survie submerge toute réflexion sur la signification de l'expérience vécue. Pour le narrateur, l'émotivité en face des épisodes les plus bouleversants se limite à une indignation laconique (KOUROUMA, $2000: 121$ ) : "Je pleurais pour leurs mères. Je pleurais pour tout ce qu'ils n'ont pas vécu ». La violence foncière du cadre narratif se combine avec l'exactitude des parenthèses historiques qui servent de coulisse à l'action, si bien que le narrateur cède à la tentation d'associer le temps de la narration à celui de la réalité historique.

De par la configuration séquentielle du récit, il est manifeste que le narrateur relate ses expériences de la guerre civile en Sierra Leone et au Libéria juste après sa rencontre avec le docteur Mamadou Doumbia. La proximité temporelle entre le moment de l'action et le temps de la narration explique sans doute la forte empreinte du vécu sur la psychologie du narrateur. En dehors d'une évocation laconique de ses forfaits (KOUROUMA, 2000 : 11) "j’ai tué beaucoup de gens avec kalachnikov (ou kalach) [...]», le jeune Birahima pourrait parfaitement passer pour un enfant innocent.

Pour éviter de raviver les plaies psychiques éventuelles, le regard du narrateur survole généralement le théâtre des opérations militaires et s'attarde uniquement sur les enfants morts au combat. Par contre, il fait rarement cas du sort des soldats adultes. Aux yeux du protagoniste pourtant habitué aux horreurs des exactions, le tableau de la guerre tribale frise l'ineffable. Certes, l'implication du narrateur qui présente un récit à la première personne permet d'exposer au lecteur ses états d'âme, mais le récit ne s'y attarde guère et la densité du ressort dramatique occulte les allusions aux ressentiments du narrateur. En outre, cette focalisation favorise le décloisonnement du temps du récit (GENETTE, 1972 : 106) : "Le récit 'à la première personne' se prête mieux qu'aucun autre à l'anticipation, du fait même de son caractère rétrospectif 
déclaré, qui autorise le narrateur à des allusions à l'avenir, et particulièrement à sa situation présente, qui font en quelque sorte partie de son rôle».

Le héros de Kourouma ne manque aucune occasion d'insérer d'autres épisodes dans le temps raconté. Les digressions historiques mettent en lumière les dérives des pouvoirs politiques en Afrique postcoloniale et le décor chaotique de l'action est présenté comme la conséquence logique des turpitudes des hommes politiques. Kourouma fait ainsi écho à Frantz Fanon qui constate dans son ouvrage Les damnés de la terre:

C'est à la fois la misère du peuple, l'enrichissement désordonné de la caste bourgeoise, son mépris étalé pour le reste de la nation qui vont durcir les réflexions et les attitudes.

Mais les menaces qui éclosent vont entraîner le raffermissement de l'autorité et l'apparition de la dictature. Le leader [...], parce qu'il cautionne l'entreprise de cette caste et ferme les yeux sur l'insolence, la médiocrité et l'immoralité foncière de ces bourgeois, constitue un écran entre le peuple et la bourgeoisie rapace. (FANON, $1968: 110$ )

La somme des inconséquences politiques aboutit au processus d'implosion sociopolitique qui alimente le phénomène des enfants- soldats. À la différence de l'étoffe classique de l'enfant soldat qui est enrôlé de force après l'assassinat des parents ou pour protéger son groupe ethnique, l'enfant soldat de Kourouma refuse de trouver au combat qu'il mène une quelconque motivation tribale ou idéologique. Il s'engage souvent dans les groupes armés pour trouver sa subsistance.

Le narrateur exerce un contrôle total sur l'action et sélectionne les segments à livrer au lecteur. Par ce biais, il contrôle la réceptivité du lecteur, brise l'élan de la narration en agitant de manière ostentatoire les indices fictionnels. La fonctionnalité des différents segments narratifs insérés dans le ressort dramatique se renforce au fil des nombreuses histoires personnelles qui retardent le dénouement de l'action. Ces histoires surviennent souvent après des séquences relatant des confrontations militaires soldées par la mort d'enfants-soldats. Elles sont parfois présentées par le narrateur comme une oraison funèbre à la gloire des enfants tombés sur le front (KOUROUMA, 2000 : 100): "Comme Kik devait mourir, était déjà mort, il fallait faire son oraison funèbre ». Les oraisons funèbres partent d'une rétrospective sur le parcours existentiel de l'enfant soldat, les circonstances de son enrôlement et sa carrière d'enfant-soldat.

De ce fait, le temps raconté dévie de celui de la narration lorsque la situation de deuil provoque chez le narrateur une réminiscence des histoires que les victimes lui auraient racontées. La récurrence de ces épisodes homodiégétiques dispense le narrateur de s'attarder sur les effets dévastateurs des moments succédant à la tension 
du combat sur son mental. La diversion est toute trouvée, lorsque le récit quitte le champ chronologique de la narration pour se focaliser sur un rétrospectif partant d'un axe temporel révolu avant de remonter vers le temps raconté. Birahima se retrouve donc au cœur d'une constellation d'histoires personnelles très émouvantes.

Du reste, le recours à des segments narratifs relatant les histoires d'enfants soldats tombés au front peut avoir une fonction cathartique, car ces épisodes interrompent les instants d'émotion où le narrateur s'apitoie de la mort de ses camarades. La profusion des épisodes intradiégétiques fait du récit de Birahima une matrice narrative contenant des histoires d'enfants-soldats morts sur le front des guerres civiles libériennes et sierra léonaises et d'autres histoires.

S'agissant des enfants-soldats, leur histoire se présente généralement sous une forme nécrologique. Car la plupart des enfants - soldats dont le parcours existentiel est évoqué sont fauchés par la mort. La mort est présente à toutes les étapes de la narration, si bien que le narrateur se sent obligé de la nourrir de l'histoire d'autres personnages pour que la sienne continue. Le décor prend l'allure d'une scène virtuelle où l'entrée de chaque acteur engendre un détour, ce qui n'est pas sans rappeler le procédé du genre dramatique. Genette souligne l'influence exercée par le genre dramatique sur la pratique narrative :

On ne doit pas méconnaître l'influence exercée pendant des siècles, sur l'évolution des genres narratifs, par ce privilège accordé à la diction dramatique. Il ne se traduit pas seulement par la canonisation de la tragédie comme genre suprême dans toute tradition classique, mais aussi, plus subtilement et bien au-delà du classicisme, dans cette sorte de tutelle exercée sur le narratif par le modèle dramatique qui se traduit si bien dans l'emploi du mot « scène » pour désigner la forme fondamentale de la narration romanesque. (GENETTE, 1972 : 193)

En scrutant de près les mécanismes du récit dans Allah n'est pas obligé, on distingue quatre axes actes narratifs distincts : un axe transversal qui ressasse les randonnées périlleuses du protagoniste, un axe explicatif qui présente l'histoire des Etats en situation de désintégration sociopolitique et deux axes biographiques. Les axes biographiques concernent justement les récits de la vie d'enfants-soldats morts à la guerre et ceux de chefs de guerre sous la férule desquels le jeune protagoniste se met pour combattre. L'axe transversal est le récit majeur dont le fil se déroule aux yeux du lecteur à mesure que progresse la narration. Il se présente de manière mathématique comme un axe des abscisses vers lequel convergent l'axe explicatif et les deux autres axes biographiques. Ainsi, le récit étend sa trame continue que croisent à des points divers les axes explicatifs et biographiques. La con- 
vergence esthétique entre ces axes du récit réside dans le fait qu'ils procèdent toutes d'histoires, soit personnelles soit nationales.

Les histoires s'enchevêtrent, le récit de la vie de Birahima est parfois interrompu par de longs détours explicatifs qui décrivent le parcours des pays en guerre depuis les indépendances jusqu'à l'avènement de la guerre civile. Celle-ci vient généralement plonger les pays dans un chaos abyssal succédant aux turpitudes antérieures d'hommes d'Etats corrompus et espiègles. Se retrouve inséré dans le récit, le leitmotiv des dictateurs africains qui traversent en partie l'œuvre de Kourouma de Les soleils des indépendances à En attendant le vote des bêtes sauvages. L'image générique utilisée par Kourouma pour dépeindre les dictateurs est présentée de la manière suivante :

Saisis dans la dynamique des relations néocoloniales où les puissances étrangères « supervisent » le naufrage des états africains dans l'anarchie politique, le confusionnisme idéologique et les droits de l'homme confisqués au profit des Guides [...], les dictateurs de Kourouma ont ceci de particulier qu'ils se ressemblent comme deux gouttes d'eau en dépit de leur naissance, leur religion, leur éducation, leur idéologie, leur vécu quotidien. (KAZI-TANI, 2001 : 335)

Le degré de vraisemblance de ces séries explicatives avec la vérité factuelle se conforte à mesure qu'apparaissent dans le récit des histoires impliquant des personnages ayant vécu dans la réalité. Ainsi, à mesure que le héros fuit la morbidité isotopique des images pour se réfugier dans des détails historiques, le lecteur averti des connexions géopolitiques de l'Afrique de l'Ouest reste enchaîné à une réalité fictionnelle confortée par l'entrée en jeu des personnages historiques réels (KOUROUMA, 2000 : 108): “[...] Ils s'étaient enfuis en Côte d'Ivoire et, là avaient pleuré auprès du dictateur du pays, Houphouët- Boigny. HouphouëtBoigny les avait consolés et les avait envoyés au dictateur de Lybie le seigneur Kadhafi qui a toujours un camp pour former les terroristes». Et plus loin dans le roman, la fiction évoque un autre homme d'Etat factuel :

Les explications se trouvent dans les jalousies entre deux dictateurs : le dictateur Houphouët- Boigny et le dictateur Sani Abacha. C'étaient les troupes de Sani Abacha qui se battaient en Sierra Léone et c'était chez Houphouët- Boigny que se tenaient les pourparlers de paix. C'étaient les compatriotes de Sani Abacha qui mouraient en Sierra-Leone et c'était de Houphouët qu'on parlait dans les journaux internationaux ; c'était lui qui était considéré comme le sage de l'Afrique noire. (KOUROUMA, $2000: 183$ ) 
On observe donc une interruption de la fiction qui se perd dans les enchevêtrements géopolitiques de la sous-région ouest africaine. Alors que les hommes d'Etat des pays déchirés par les guerres tribales atroces s'intègrent directement dans le flux du récit, les hommes d'Etats externes font l'objet de détours par lesquels les connexions exogènes des guerres civiles ouest-africaines sont élucidées avec une description presque burlesque des acteurs externes. De manière insidieuse, le roman identifie alors les fauteurs proches et lointains du décor dramatique et chaotique où Birahima déploie sa narration. La responsabilité incombe aux dictateurs externes qui agissent par souci de reconnaissance et de prestige sous- régional et aux dictateurs internes dont l'appétence du pouvoir fait naître des séditions militaires :

Dans ces pays pauvres, sous-développés, où, selon la règle, la plus grande richesse côtoie la plus grande misère, l'armée et la police constituent les piliers du régime. Une armée et une police qui, encore une règle dont il faudra se souvenir, sont conseillées par des experts étrangers. La force de cette police, la puissance de cette armée sont proportionnelles au marasme dans lequel baigne le reste de la nation. (FANON, $1968: 113$ )

Dans Allah n'est pas obligé, la connexion se fait aisément avec l'histoire littéraire puisque le destin de Birahima est une conséquence logique de la dérive des dictatures africaines dénoncée dans le premier roman de Kourouma Les soleils des indépendances. Du reste, le roman Allah n'est pas obligé est la maturation chez Kourouma d'un procédé stylistique qui démystifie la langue du colonisateur et la « colonise ». Une telle pratique linguistique et stylistique répond parfaitement à la conception du roman, si bien que l'étoffe du protagoniste et narrateur semble dériver d'un désir de se « jouer » de la langue. Ainsi en mettant en scène un personnage imparfaitement hybride du point de vue linguistique, les éléments du jeu sont en place. Du coup, la progression du récit est souvent interrompue de parenthèses métalexicales. La fuite de la réalité du front est alors avant tout une question de mots qui brisent le flot du récit et donne au protagoniste, la possibilité de manipuler librement la focalisation de la narration.

\section{PARAVENTS PSYCHOLOGIQUES :}

L'originalité du récit de Birahima réside avant tout dans la tour de force lexicale que constitue le recours à des parenthèses d'explication de mots au cœur même de la narration. Au risque d'irriter le lecteur, la rupture de l'élan narratif joue un rôle déterminant dans l'organisation du décor fictif. Le récit étant structuré autour de la vie du narrateur déployé dans un univers où les coulisses de 
l'action ont une ressemblance trop marquante avec la réalité, il est impérieux pour la fiction de remettre en cause les indices factuels. A cela servent les nombreuses interruptions induites par les questions lexicales ou stylistiques. Mais le lecteur est averti à l'avance puisque Birahima lui confie (KOUROUMA, 2000 : 11) : "Pour raconter ma vie de merde, de bordel de vie dans un parler approximatif, un français passable, pour ne pas mélanger les pédales dans les gros mots, je possède quatre dictionnaires ".

Naît dès lors une sorte de contrat entre le narrateur et le lecteur contraint de jouer le jeu. Seule la forme romanesque donne à l'énonciateur cette possibilité d'une adresse directe ou indirecte. Pourtant, cette même forme est conditionnée par une prise en compte de la psychologie des personnages. Et comme Birahima a choisi de présenter sa propre histoire, il se trouve exposé à révéler la trame de son psychisme devant cette litanie terrifiante de meurtres, de massacres, d'assassinats et de cruautés. Le protagoniste y échappe en mettant en exergue les enjeux de l'hybridité linguistique. Les formes stylistiques du récit présenté par le narrateur place la transgression de la langue française à son paroxysme. Le programme originel de Kourouma est esquissé de la manière suivante (BOURGMANO, 2001: 343): "Ahmadou Kourouma, désireux de montrer que la mainmise linguistique sur l'Afrique, bien plus sournoise que l'oppression économique et politique, lui était pourtant inextricablement liée, a fait de la traduction-transposition à la fois le thème central de ses romans et le ressort essentiel de son écriture ».

Mais dans Allah n'est pas obligé, la transgression atteint son paroxysme comme pour conjurer la violence inouïe des images agitées par le narrateur. Même l'instrument linguistique échoue à altérer les chocs dérivant de cette atmosphère foncière d'atrocité inhumaine. L'étoffe du protagoniste, la focalisation et l'énoncé du récit entretiennent une collusion avec la déviance linguistique. La particularité du vecteur psycholinguistique est patente et Borgmano de constater :

Mais Allah n'est pas obligé opère, par rapport aux précédents romans, une inversion radicale. Il quitte le point de vue des puissants et adopte celui des défavorisés, des plus dépourvus de pouvoirs, les « enfants des rues ». La traduction reste une question centrale, mais elle se pose tout autrement, et d'une certaine façon à l'envers, aussi bien au niveau du discours du roman qu'au niveau diégétique. (BOURGMANO, 2001:345-346)

Le profil du protagoniste correspond parfaitement au tour de force linguistique que veut réaliser Kourouma. La violence isotopique du décor fictif contamine la langue du narrateur qui viole systématiquement tous les codes linguistiques. En effet, la désintégration de l'appareil social et politique dans les pays servant de décor au récit se cristallise dans une pratique linguistique dissolue. Sans doute que 
l'élément linguistique est le meilleur illustrateur des expériences de la guerre sur la mémoire personnelle du narrateur. Pour éviter de devoir dire avec des termes crus les atrocités inimaginables des groupes armés, l'auteur passe par la dérision linguistique et le burlesque. La matrice stylistique de ces genres de récit trouve l'adhésion de Kourouma qui l’intègre à sa transgression de la langue française. Le résultat est une forte empreinte de l'élément psycholinguistique si bien que le narrateur même l'évoque directement dans son récit.

Le lecteur en subit les interférences intempestives et le flux de la narration est interrompu de manière impromptue par la profusion des parenthèses lexicales et sémantiques. Celles-ci disloquent le récit et permettent, en l'espace de quelques lignes, de détourner le regard de la cruauté foncière des acteurs. Les conditions sont dès lors réunies pour que le protagoniste se réfugie derrière cette anarchie linguistique et soustrait sa psychologie au chaos ambiant. L'expérience de la guerre devient un prétexte pour le narrateur qui s'autorise un vocabulaire parfois abject. Voici comment Daniel Delas commente l'excentricité lexicale du roman:

Peu à peu se fait jour dans la compétence lecturale de vous et moi le soupçon que la justification avancée au début du livre - ce recours à des définitions de dictionnaires a pour fonction de permettre à divers types de lecteurs d'accéder à la compréhension du texte - est de pure convention. Un peu comme une couverture socio-linguistique politiquement correcte d'une poétique décapante, fondée sur l'installation d'une sorte de chambre d'échos, où chaque mot important résonne, amplifié jusqu'à l'absurde, décrédibilisant la valeur des mots. (DELAS, $2001:$ 378)

La trivialité du ressort linguistique, la récurrence des proverbes ${ }^{4}$ et des interjections à la limite de la décence verbale occupent trop bien le lecteur pour qu'il scrute les effets de la guerre sur le psychisme du protagoniste. Fouiller les dictionnaires, expliquer les "gros mots » malinkés occupe suffisamment le narrateur. Captivé par l'histoire et les relents humoristiques de la narration, le lecteur accepte ce «manque de sérieux » et la forte empreinte de la dérision qui rend inopérante toute référence à la psychologie du narrateur. La langue devient pour lui un refuge, " les gros mots » une sorte de remède cathartique contre l'omniprésence de la mort. Delas (2001: 378) souligne que «la langue s'est ainsi déréalisée au profit du rythme du discours qui porte, par toutes les formes d'écho métalexicographique qu’il met en place, la signifiance profonde de l'œuvre ».

\footnotetext{
${ }^{4}$ La fonction des proverbes chez Kourouma est commentée par Nora- et Alexandra Kazi-Tani (2001 : 338) : "loin de jouer simplement un rôle esthétique, loin d'être simplement des clins d'œil culturels, ils fonctionnent comme des éléments phosphorescents, synthèses saisissantes après une séquence analeptique ou motifs anaphoriques d'une idée motrice ".
} 
Les refuges psycholinguistiques sont constitués soit par les proverbes, soit par les «gros mots de toute sorte» que le narrateur tient à expliquer. Venant de Kourouma dont les approches de l'élément psycholinguistique sont très complexes, cela n'est vraiment pas étonnant. Mais le lecteur est forcément surpris par cette sollicitude et l'irruption intempestive d'ouvrages nantis d'une autorité lexicale incontestable induit une transfrontalité dérangeante. On passe ainsi d'un monde narratif foisonnant à une constellation de gloses sémantiques qui entrecoupent l'élan du récit. Même si la défiance stylistique de l'auteur à l'endroit de la « langue du colonisateur » est connue, les entrelacements entre les éléments factuels et les instruments de glose sémantique présentent un risque énorme dans la réception, puisque les digressions lexicales peuvent ennuyer voire irriter le lecteur.

La langue étant un enjeu central de l'esthétique romanesque d'Ahmadou Kourouma, nul peut cerner le problème de manière expéditive en expliquant l'usage des gloses comme résultant des circonstances de la narration. Au contraire, les dictionnaires fonctionnent comme un refuge où s'abrite le narrateur contre l'énorme tension suscitée par les mots. Les multiples explications occupent le lecteur qui peut se passer de toute digression supplémentaire censée lui livrer les sentiments du narrateur. Or le récit à la première personne rend incontournable la référence aux états d'âme du narrateur. L'instance narrative fuit et se réfugie derrière les mots puisque la question psycholinguistique occupe le devant du récit. Les effets psychosomatiques de la violence sur le narrateur se manifestent par un agencement labile de la trame linguistique qui subit les contrecoups de cette violence. Tout comme la guerre déchire le tissu socioéconomique et politique, de même la langue est contaminée par le chaos ambiant. Du coup, la trame stylistique tient un rôle central dans l'organisation du cadre narratif. Les effets dévastateurs du ressort dramatique sont répercutés sur la tenure langagière du roman. Les autres personnages parlent et agissent au travers des mots de Birahima, dont l'énoncé dérive d'un réseau d'anomies sociales, politiques et lexicales.

L'évolution des différents épisodes du récit est seulement retardée par le foisonnement lexical volontairement orchestré par l'auteur. En cédant au narrateur le contrôle total sur toute l'évolution du récit, Kourouma lui laisse l'occasion de relater des événements géopolitiques complexes dans un lexique assez élémentaire pour être compris de tous. Les mots sont sa seule arme contre l'empreinte des atrocités vécues sur son devenir, il déverse sur la transgression lexicale des codes sémiotiques la violence ressentie au plus profond de son être. Le mot devient le réceptacle de ses traumatismes dissimulés sous la fluidité du récit dont la lumière éclaire seulement de manière lapidaire les états d'âmes du protagoniste. Mais en revenant aux sources même du récit, on peut se rendre compte que la stratégie narrative répond parfaitement aux stéréotypes de l'enfant autant dans la fiction romanesque que dans la réalité de la guerre. Même si les enfants se glorifient des actes de guerre héroïques et des massacres commis, ils sont toujours perçus comme des victimes. 
Même si le protagoniste de Kourouma relate avec une lucidité froide les forfaits des chefs de guerre et des combattants, il passe dans la fiction romanesque comme un enfant manipulé qui ne saurait réfléchir à la signification de la guerre et des atrocités. Par conséquent, insérer des séquences réflexives dans le récit du personnage reviendrait à lui conférer une conscience assez forte des évènements, à engager ses responsabilités. Or, un tel modèle d'enfant-soldat n'est ni acceptable pour les stéréotypes fictifs ou factuels existants, car il semble conforter seulement le rôle de bourreau des enfants armés par les adultes. Faire dire à un enfant qu'il a tué, violé, découpé ses semblables est un passif lourd que la bienséance artistique réfrène. C'est à juste titre que l'enfant-soldat reste dans les coulisses de l'action et prend une posture d'observateur lorsqu'il s'agit de raconter des actions coupables. Birahima reconnaît certes avoir (KOUROUMA, 2000 : 11) « tué beaucoup de gens au Libéria et en Sierra-Leone ", mais n’indique à aucun moment du récit les détails et les circonstances de ses forfaits.

Une présentation aussi superficielle de l'implication du héros constitue sans aucun doute une fuite patente de la réalité. Si la forme romanesque offre par essence la possibilité d'accéder au psychisme des acteurs, la focalisation relativise cette possibilité. La fonction du narrateur est présentée par Anne Maurel de la manière suivante:

Le roman ne nous met jamais en présence de faits bruts. Le romancier fait le choix des faits qu'il retiendra, de ceux qu'il mettra en relief, il choisit aussi de nous en donner une connaissance objective ou subjective, partielle ou totale, interne ou externe [...]. Car le narrateur joue un rôle capital dans la construction de la fiction, puisqu'il décide du temps du récit et du ou des points de vue sur les faits. Il peut en effet raconter les évènements dans l'ordre dans lequel ils ont eu lieu, ou bouleverser la chronologie réelle ou fictive par toutes sortes d'achronies, par des retours en arrière, ou des annonces de la suite [...]. C'est le narrateur encore, qui selon qu'il adopte sur les faits et les personnages du roman un point de vue omniscient, ou au contraire qu'il restreint sa vision à la perspective forcément limitée d'un personnage de l'histoire, nous donne du monde raconté une connaissance totale ou partielle, objective ou subjective. (MAUREL, 1998 : 82-83)

Puisque la présence du narrateur au cœur du récit ne peut s'accommoder d'une description des mécanismes réflexifs chez les personnages, le lecteur doit se contenter d'une fenêtre éclairant les états affectifs du narrateur. Ce dernier prend la posture d'un observateur qui prête son regard au lecteur sur les champs de bataille des guerres civiles ouest-africaines. Cette perspective lui évite de se trouver à la loupe de 
la narration dont il se refuse à être le référent. Le protagoniste refoule systématiquement les stigmates du traumatisme vécu et il est clair que l'étoffe mentale d'un jeune enfant presque analphabète ne peut affronter la cruauté foncière des acteurs.

Les personnages ont une vie intérieure labile et l'instinct de conservation les confine dans la sphère matérielle et superficielle de l'existence. Miné par un effondrement de toutes les valeurs, le décor du récit réfrène toute activité de l'esprit et le moi cède facilement à la violence incommensurable des pulsions. Même le narrateur accepte le sceau sanglant de la violence sur son psychisme et il se plaît à assimiler les évènements sans s'interroger sur leur signification. Daniel Delas (2001: 370) essaie de cerner le problème par des interrogations suivantes: "Avoir dix ans, une kalachnikov, des uniformes trop grands et être jetés dans un maelstrom de fusillades, embuscades, guet-apens, exécutions, pillages, massacres en tout genre, comment cela se vit-il ? Comment ce réel incroyable peut-il être dit ou à tout le moins évoqué de manière crédible »?

$\mathrm{Au}$ demeurant, l'âge du narrateur et sa courte expérience scolaire ne lui donnent pas les moyens de structurer sa mémoire personnelle autour des épisodes soigneusement sélectionnés par son psychisme. Il procède par un processus de cloisonnement de son intériorité qui semble échapper à l'empreinte ruineuse de la violence aveugle. Birahima passe pour un personnage apathique puisque les épisodes les plus cruciaux du ressort dramatique l'impliquent rarement. Ce ressort est habilement distendu par le parcours de la tante Mahan dont la recherche devient une quête de la vérité littéraire sur les guerres civiles libériennes et sierra-léonaises. Mais, cette vérité est si poignante que les mots ordinaires ne peuvent le dire.

Du coup, c'est dans le langage infiniment désaxé du narrateur qu'il faut saisir cette vérité. Aucune poétique classique ne peut rendre compte de ce fol enchaînement de situations anomiques auquel est confronté le protagoniste. Il transpose son malaise sur les mots et les lance à la figure du lecteur, dans un désir d'exorciser verbalement les images terribles qui hantent son imaginaire. Delas (2001:371) évoque le problème de manière lapidaire : «Comment parle un enfant africain » ? Birahima ne raconte pas, il crache les mots, désamorce la tension grâce aux dictionnaires et replonge à nouveau le lecteur dans un ferment lexical situé au-delà des limites de la décence. Les « Faforo », « Gnamokodé » et «Walahé » sont le reflet du désordre psychique qui grouille dans la tête du protagoniste.

\section{CONCLUSION :}

La formulation de la vérité sur les guerres tribales d'Afrique subsaharienne par Kourouma laisse transparaître chez l'auteur des biais qui visent à tempérer la violence des images. Avec un jeune garçon simpliste au centre de la narration, se précise la problématique du langage juste pouvant décrire l'absurde frivolité des personnages qui rivalisent de cruauté. L'univers de la narration est parsemé 
d'évènements fantasques et crus pour les yeux d'un gamin de l'âge de Birahima. Le résultat est un flot narratif entrecoupé de parenthèses historiques très proches de la réalité et semé de clins d'œil burlesques. La densité des références factuelles oblige l'auteur à structurer le récit autour d'éléments rappelant sans cesse au lecteur le caractère fictif des événements relatés.

Dès lors, les séquences de la narration et le fil dramatique sont articulés autour d'une fuite : fuite linguistique qui dispense le narrateur d'utiliser les mots sérieux susceptibles de toucher l'émotivité du lecteur; fuite historique qui intègre dans la narration l'évolution des nations indépendantes de l'Afrique de l'Ouest. Une préoccupation centrale semble concentrer les techniques narratives investies par l'auteur: comment garantir la survie mentale du jeune protagoniste dont la bouche laisse jaillir des mots d'une gravité démesurée ? Et la réponse de Kourouma semble être une violence langagière du héros, en guise de réaction à la violence psychique résultant des fresques macabres vues par ses yeux d'enfants. En violentant le code linguistique et le code social de son milieu d'existence, le protagoniste refoule aisément son malaise.

\section{REFERENCES BIBLIOGRAPHIQUES}

BOURGMANO, M. Kourouma et les gros mots. In: LITTERATURES AFRICAINES : TRANSPOSITONS ? Carnets du CERPANAC $\left(\mathrm{n}^{\circ} 2\right)$, 2001. Université de Montpellier III : APELA, (pp343- 354).

DELAS, D. Rythmes et parenthèses dans Allah n'est pas obligé d'Ahmadou Kourouma. LITTERATURES AFRICAINES: TRANSPOSITONS? Carnets du CERPANAC $\left(\mathrm{n}^{\circ}\right.$ 2), 2001. Université de Montpellier III : APELA, (pp 369-380).

FANON, F. Les damnés de la terre. Paris : Maspéro, 1968.

GENETTE, G. Figures III. Paris : Seuil, 1972.

KAZI- TANI, Nora et Alexandra : L'écriture à l'intersection des signes dans le roman d'Ahmadou Kourouma En attendant le vote des bêtes sauvages. LITTERATURES AFRICAINES : TRANSPOSITONS ? Carnets du CERPANAC $\left(\mathrm{n}^{\circ}\right.$ 2), 2001. Université de Montpellier III : APELA, (pp 329-342).

KOUROUMA, A. Allah n'est pas obligé. Paris : Seuil, 2000.

MAUREL, A. La critique. Paris : Hachette, 1998. 